\title{
Decay of weak solutions and the singular set of the three-dimensional Navier-Stokes equations
}

\author{
James C. Robinson \\ Mathematics Institute, University of Warwick, Coventry CV4 7AL, UK \\ E-mail: j.c.robinson@warwick.ac.uk \\ Witold Sadowski \\ ${ }^{1}$ Faculty of Mathematics, Informatics and Mechanics, Warsaw University, Banacha 2 \\ 02-097, Warszawa, Poland. \\ ${ }^{2}$ Institute of Mathematics of the Polish Academy of Sciences, ul. Śniadeckich 8 \\ 00-956, Warszawa, Poland. \\ E-mail: witeks@hydra.mimuw.edu.pl
}

\begin{abstract}
We consider the behaviour of weak solutions of the unforced threedimensional Navier-Stokes equations, under the assumption that the initial condition has finite energy $\left(\|u\|^{2}=\int|u|^{2}\right)$ but infinite enstrophy $\left(\|D u\|^{2}=\int|\operatorname{curl} u|^{2}\right)$. We show that this has to be reflected in the solution for small times, so that in particular $\|D u(t)\| \rightarrow+\infty$ as $t \rightarrow 0$. We also give some limitations on this 'backwards blowup', and give an elementary proof that the upper box-counting dimension of the set of singular times can be no larger than one half. Although similar in flavour, this final result neither implies nor is implied by Scheffer's result that the 1/2-dimensional Hausdorff measure of the singular set is zero.
\end{abstract}

Submitted to: Nonlinearity 


\section{Introduction}

The existence or otherwise of singularities in the three-dimensional Navier-Stokes equations is acknowledged to be one of the outstanding open problems in the theory of partial differential equations, and so there has been much research concerned with the time before the formation of a possible singularity. In this paper we consider the behaviour of solutions after the occurrence of a singularity, or equivalently (but less hypothetically) the time evolution of a weak solution with a suitably irregular initial condition.

In order to describe our results in more detail we need some simple terminology and notation. Throughout the paper we consider the evolution of an incompressible fluid in a periodic box $\Omega=[0, L]^{3}$ in the absence of external forces: the evolution of the velocity field $u(x)$ is modelled by the three-dimensional Navier-Stokes equations

$$
\frac{\partial u}{\partial t}-\nu \Delta u+(u \cdot \nabla) u+\nabla p=0 \quad \nabla \cdot u=0
$$

For convenience sake we also assume that the total momentum is zero, $\int_{\Omega} u(x) \mathrm{d} x=0$, a situation that is preserved under the evolution of $u$.

We denote the kinetic energy of $u(x)$ by

$$
\|u\|^{2}:=\int_{\Omega}|u(x)|^{2} \mathrm{~d} x
$$

and the 'enstrophy' of $u(x)$, which is the square integral of the vorticity $\omega(x)=\operatorname{curl} u(x)$, by

$$
\|D u\|^{2}:=\int_{\Omega}|\operatorname{curl} u(x)|^{2} \mathrm{~d} x .
$$

We denote the space of all divergence-free flow fields with finite kinetic energy by $H$, and the space of all such flow fields with finite enstrophy by $V$. (More strictly, if $\mathscr{V}$ is the space of all $C^{\infty} \Omega$-periodic functions that are divergence free and have zero average over $\Omega$, then $H$ is the closure of $\mathscr{V}$ in the $L^{2}$ norm, and $V$ is the closure of $\mathscr{V}$ in the $H^{1}$ norm.)

Leray showed in 1934 that given an initial condition with finite energy, there is a weak solution of the equations that exists for all time and whose kinetic energy remains finite. Indeed, we have

$$
\|u(t)\|^{2}+\int_{0}^{t}\|D u(s)\|^{2} \mathrm{~d} s \leq\left\|u_{0}\right\|^{2}
$$

for all $t \geq 0$, and the solution is weakly continuous into $H$, i.e. $u(t) \rightarrow u(s)$ as $t \rightarrow s$. However, the uniqueness of such a solution is not guaranteed.

On the other hand, while it is know that if the enstrophy $\|D u(t)\|^{2}$ remains finite then the solution is unique, an initial condition $u_{0}$ with $\left\|D u_{0}\right\|^{2}<\infty$ is only known to give rise to a solution whose enstrophy remains finite for a limited time. [For an elementary discussion of these issues see Robinson (2006); for a more advanced but 
accessible treatment see Doering \& Gibbon; and for a more technical mathematical discussion see Constantin \& Foias (1988) or Temam (1977).]

The possibility of singular behaviour in the Navier-Stokes equations was first pointed out by Leray (1934) who suspected the existence of self-similar singular solutions. He also showed (using a simple argument which we will review later) that any solution that blows up at a time $t=T$ must be bounded below by

$$
\|D u(t)\|^{2} \geq \frac{c}{\sqrt{T-t}}
$$

Still little is known of the regularity (or otherwise) of weak solutions of the NavierStokes equations, but several papers have imposed some restrictions on the hypothetical blow up. The most famous is probably Scheffer's result stating that the Hausdorff measure of turbulent times in the Navier-Stokes equations is less than one half. More sophisticated is the result of Caffarelli, Kohn, \& Nirenberg (1982), which treats the singular set in space-time (i.e. as a subset of $\Omega \times[0, \infty)$ ). Scheffer's result can be obtained as a consequence of the result of Caffarelli et al., see Section 7 in Robinson (2006).

Here we give a simple proof of a result reminiscent of Scheffer's: we show that the upper box-counting dimension of the singular set is no greater than $1 / 2$. This result neither implies, nor is implied by, Scheffer's.

\section{Solutions with irregular initial data}

Given initial data $u_{0} \in V$, it is well known that the solution remains regular (i.e. $\|D u(t)\|$ remains finite) at least for some short time interval. Indeed, standard estimates can be used to show that for $t>s$

$$
\|D u(t)\|^{2} \leq \frac{\|D u(s)\|^{2}}{\sqrt{1-c(t-s)\|D u(s)\|^{4}}}
$$

while the right-hand side remains finite, i.e. while

$$
c(t-s)\|D u(s)\|^{4}<1 .
$$

In particular, if $s=0$ then this shows that the solution remains regular at for $t \in[0, T)$, where $T=c^{-1}\left\|D u_{0}\right\|^{-4}$.

Rearranging (1) to obtain a lower bound for $s<t$ gives

$$
\|D u(s)\|^{2} \geq \frac{\|D u(t)\|^{2}}{\sqrt{1+c(t-s)\|D u(t)\|^{4}}} .
$$

While one might be concerned as to the range of validity of this bound (given the conditions on $t$ and $s$ in (2), it does in fact hold for all $s \leq t$. Indeed, if for some $s<t$ one has

$$
\|D u(s)\|^{2}<\frac{\|D u(t)\|^{2}}{\sqrt{1+c(t-s)\|D u(t)\|^{4}}},
$$


then the right-hand side of (1) remains finite on $(s, t]$ and one can infer that $\|D u(t)\|^{2}<$ $\|D u(t)\|^{2}$, a contradiction.

From (3) one can easily deduce (as did Leray) that if a solution does blow up as $t \rightarrow T$, i.e. if $\|D u(t)\| \rightarrow \infty$ as $t \rightarrow T$, then for $s<T$ one must have the lower bound

$$
\|D u(s)\|^{2} \geq \frac{1}{\sqrt{c(T-s)}} \quad \text { for all } \quad 0 \leq s<T .
$$

Given $u_{0} \in V$, we know that even if there is a loss of regularity at some time $T$, there is a weak solution that exists for all positive times. It is therefore reasonable to ask about the behaviour of this solution after the putative blowup time. Even the equations are in fact 'regular' - i.e. if any initial condition in $V$ gives rise to a regular solutions that is valid for all time - one can still ask the same question about solutions whose initial condition is contained in $H$ and not in $V$.

Our first simple result shows that such solutions retain a trace of this 'irregularity', i.e. it is not possible that $\left\|D u_{0}\right\|=\infty$ but $\|D u(t)\|$ is uniformly bounded on an interval of the form $(0, \epsilon)$ for some $\epsilon>0$.

Lemma 1.1. Suppose that $u_{0} \in H \backslash V$, and that $u(t)$ is a weak solution with $u(0)=u_{0}$. Then $\|D u(t)\| \rightarrow+\infty$ as $t \rightarrow 0$.

Proof. Suppose not. Then for some $M>0$ we have a sequence $t_{n}$ with $\left\|D u\left(t_{n}\right)\right\| \leq M$. Since $u$ is weakly continuous into $H$, we know that $u\left(t_{n}\right) \rightarrow u_{0}$ in $H$. However, since $\left\|D u\left(t_{n}\right)\right\| \leq M$ it follows from the Alaoglu Theorem that $u\left(t_{n}\right) \rightarrow v_{0}$ in $V$, for some $v_{0}$ with $\left\|D v_{0}\right\| \leq M$. Since weak convergence in $V$ implies weak convergence in $H$, and weak limits are unique, we must in fact have $u_{0}=v_{0}$, and so $\left\|D u_{0}\right\| \leq M$, a contradiction.

\section{Decay of solutions}

Given the result of this lemma, it becomes natural to look for possible upper or lower bounds on the rate of decay of the $V$ norm for solutions with irregular initial data, and we now turn to some results in this direction.

All of our results will be consequences of the lower bound in (3), which is more convenient to work with when written in the form

$$
\|D u(s)\|^{2} \geq \frac{1}{\sqrt{\|D u(t)\|^{-4}+c(t-s)}} .
$$

We note here that this lower bound remains valid even if $u$ is not regular on the interval $[s, t]$, since $\|D u(r)\|=+\infty$ for $r \in(s, t)$ implies that

$$
\|D u(s)\|^{2} \geq \frac{1}{\sqrt{c(r-s)}} \geq \frac{1}{\sqrt{\|D u(t)\|^{-4}+c(t-s)}} .
$$




\subsection{Solutions in $L^{4}(0, T ; V)$}

An immediate consequence of the inequality in (5) is an upper bound on the $V$ norm of solutions known to be in $L^{4}(0, T ; V)$. In order for

$$
\int_{0}^{T}\|D u(s)\|^{4} \mathrm{~d} s
$$

to be finite, one would expect $\|D u(t)\|^{4}$ blows up more slowly than $t^{-1}$ as $t \rightarrow 0$. In particular, it is natural to imagine that $t\|D u(t)\|^{4}$ remains bounded as $t \rightarrow 0$, and this is shown rigorously in the following result:

Proposition 2.1. Suppose that $u \in L^{4}(0, T ; V)$. Then there exists a constant $c$ depending on $\|u\|_{L^{4}(0, T ; V)}$ such that

$$
t^{1 / 2}\|D u(t)\|^{2} \leq c
$$

Proof. From (3) we have

$$
\|D u(s)\|^{4} \geq \frac{\|D u(t)\|^{4}}{1+c(t-s)\|D u(t)\|^{4}},
$$

and so

$$
\begin{aligned}
\int_{0}^{t}\|D u(s)\|^{4} \mathrm{~d} s & \geq\left[-\frac{1}{c} \log \left(1+c(t-s)\|D u(t)\|^{4}\right)\right]_{0}^{t} \\
& =\frac{1}{c} \log \left(1+c t\|D u(t)\|^{4}\right)
\end{aligned}
$$

from which (6) follows.

It is natural to conjecture that this decay also holds for all weak solutions (this is the case for the two-dimensional Navier-Stokes equations), but in general all the information we currently have available is that $u \in L^{2}(0, T ; V)$, and so a more natural conjecture along similar lines is that $t\|D u(t)\|^{2} \leq c$. However, a similar approach, but starting from (5) yields much weaker results. Indeed, if $\left\|D u\left(t_{n}\right)\right\|=+\infty$ with $t_{n} \downarrow 0$ then one can only obtain

$$
\sum_{n=1}^{\infty}\left(t_{n}-t_{n+1}\right)^{1 / 2}<\infty,
$$

an observation originally due to Leray.

\subsection{Box-counting dimension and decay of solutions}

Given a set $X$, its (upper) box-counting dimension is defined as follows. Let $N(X, \epsilon)$ denote the largest number of disjoint balls of radius $\epsilon$ with centres in $X$. Then

$$
d_{f}(X)=\limsup _{\epsilon \rightarrow 0} \frac{\log N(X, \epsilon)}{-\log \epsilon},
$$


i.e. essentially $N(X, \epsilon) \sim \epsilon^{-d_{f}(X)}$. [Usually one would take $N(X, \epsilon)$ to be the smallest number of balls of radius $\epsilon$ whose union covers $X$ (see Definitions 3.1 in Falconer, 1990), but the two definitions are equivalent and that given here is more convenient for what follows.]

We now give an indication of how the box-counting dimension occurs naturally when considering rates of decay of solutions. Suppose that

$$
\|D u(t)\|^{2} \geq c t^{-\alpha} \text {. }
$$

Then, since we know that $\int_{0}^{T}\|D u(s)\|^{2} \mathrm{~d} s \leq\left\|u_{0}\right\|^{2}$, it must be the case that $\alpha<1$. However, if (7) holds then in particular it follows that for $t_{n}=(n / c)^{-1 / \alpha}$ we have $\left\|D u\left(t_{n}\right)\right\|^{2} \geq n$. The fractal dimension of this sequence $\left\{t_{n}\right\}$ is equal to $\alpha /(1+\alpha)$, and since we should have $\alpha<1$, it follows that the dimension of this sequence should be less than one half.

Rather than proving a strong estimate such as that in (17), instead we prove that if we have a sequence $\left\|D u\left(t_{n}\right)\right\|^{2} \geq n$, then the fractal dimension of $\left\{t_{n}\right\}$ can be no larger than one half.

Proposition 2.2. Suppose that

$$
\left\|D u\left(t_{n}\right)\right\|^{2} \geq n
$$

for some sequence $t_{n} \geq 0$. Then $d_{f}\left(\left\{t_{n}\right\}\right) \leq 1 / 2$.

In the proof we write $\mathscr{T}=\left\{t_{n}\right\}$.

Proof. Suppose that $d_{f}(\mathscr{T})=d>1 / 2$. Then for some $\delta$ with $1 / 2<\delta<d$ there exists a sequence $\epsilon_{j} \rightarrow 0$ such that

$$
N_{j}:=N\left(\mathscr{T}, \epsilon_{j}\right)>\epsilon_{j}^{-\delta}
$$

where $N(\mathscr{T}, \epsilon)$ denotes the maximal number of disjoint balls centred on $t_{j}$ of radius $\epsilon$. Let the centres of these balls be $t_{n_{i}}, 1 \leq i \leq N_{j}$. Since these balls are disjoint, we have

$$
\int_{0}^{1}\|D u(s)\|^{2} \mathrm{~d} s \geq \sum_{i=1}^{N_{j}} \int_{t_{n_{i}}-\epsilon_{j}}^{t_{n_{i}}+\epsilon_{j}}\|D u(s)\|^{2} \mathrm{~d} s>\sum_{i=1}^{N_{j}} \int_{t_{n_{i}}-\epsilon_{j}}^{t_{n_{i}}}\|D u(s)\|^{2} \mathrm{~d} s .
$$

Now, using the bound in (5) it follows that

$$
\begin{aligned}
\int_{t-\epsilon}^{t}\|D u(s)\|^{2} \mathrm{~d} s & \geq \int_{t-\epsilon}^{t} \frac{1}{\sqrt{\|D u(t)\|^{-4}+c(t-s)}} \mathrm{d} s \\
& =\frac{1}{2 c}\left[-\sqrt{\|D u(t)\|^{-4}+c(t-s)}\right]_{t-\epsilon}^{t} \\
& =\frac{1}{2 c}\left[\sqrt{\|D u(t)\|^{-4}+c \epsilon}-\|D u(t)\|^{-2}\right] .
\end{aligned}
$$

Noting that $\sqrt{X^{2}+c \epsilon}-X$ is a decreasing function of $X$ we therefore have

$$
\int_{0}^{1}\|D u(s)\|^{2} \mathrm{~d} s \geq \frac{1}{2 c} \sum_{i=1}^{N_{j}}\left[\sqrt{n_{i}^{-2}+c \epsilon_{j}}-n_{i}^{-1}\right]
$$


and since $n_{i} \geq i$ it follows that

$$
\int_{0}^{1}\|D u(s)\|^{2} \mathrm{~d} s \geq \frac{1}{2 c} \sum_{n=1}^{N_{j}}\left[\sqrt{n^{-2}+c \epsilon_{j}}-n^{-1}\right] .
$$

In order to proceed we note that

$$
X<\sqrt{c \epsilon} / 2 \Rightarrow \sqrt{X^{2}+c \epsilon}-X>\frac{\sqrt{c \epsilon}}{2} .
$$

It follows, since $N_{j} \geq \epsilon_{j}^{-\delta}$ and $\delta>1 / 2$ we have, for $j$ large enough,

$$
\begin{aligned}
\int_{0}^{1}\|D u(s)\|^{2} \mathrm{~d} s & \geq \frac{1}{2 c} \sum_{n=2\left(c \epsilon_{j}\right)^{-1 / 2}}^{\epsilon_{j}^{-\delta}}\left[\sqrt{n^{-2}+c \epsilon_{j}}-n^{-1}\right] \\
& \geq \frac{1}{4 \sqrt{c}} \sum_{n=2\left(c \epsilon_{j}\right)^{-1 / 2}}^{\epsilon_{j}^{-\delta}} \sqrt{\epsilon_{j}} \\
& \geq \frac{1}{4 \sqrt{c}}\left[\epsilon_{j}^{(1 / 2)-\delta}-2 / \sqrt{c}\right] .
\end{aligned}
$$

Since $\epsilon_{j} \rightarrow 0$ as $j \rightarrow \infty$ and $\delta>1 / 2$ it follows that $\int_{0}^{1}\|D u(s)\|^{2} \mathrm{~d} s=+\infty$, a contradiction.

Our discussion above suggests that in fact one should expect to be able to prove that $d_{f}(\mathscr{T})<1 / 2$ provided one assumes in addition that $t_{n} \downarrow 0$ (an assumption not necessary for the proof of the Proposition). This is further supported by the following observation. If one has

$$
\left\|D u\left(n^{-1}\right)\right\|^{2} \geq n
$$

i.e. $t_{n}=n^{-1}$ in the statement of Proposition 2.2 , then although $d_{f}(\mathscr{T})=1 / 2$ one can show that (9) is impossible. Indeed, for all $s \in\left(t_{n+1}, t_{n}\right)$ we have

$$
\|D u(s)\|^{2} \geq \frac{\left\|D u\left(t_{n}\right)\right\|^{2}}{\sqrt{1+\left(t_{n}-s\right)\left\|D u\left(t_{n}\right)\right\|^{4}}} \geq \frac{n}{\sqrt{1+\frac{n^{2}}{n(n+1)}}} .
$$

So

$$
\int_{0}^{t_{1}}\|D u(s)\|^{2} \geq \sum_{n=1}^{\infty} \frac{1}{n(n+1)} \frac{n}{\sqrt{1+\frac{n^{2}}{n(n+1)}}} \geq \sum_{n=1}^{\infty} \frac{1}{\sqrt{2}(n+1)}=+\infty,
$$

which is a contradiction.

\section{The set of singular times}

A very similar, but slightly simpler argument, shows that if $\Sigma$ is the set of 'singular times' of a weak solution, i.e.

$$
\Sigma=\{t \geq 0: u(t) \notin V\}=\{t \geq 0:\|D u(t)\|=\infty\},
$$

then $d_{f}(\Sigma) \leq 1 / 2$ 
Corollary 3.1. The upper box-counting dimension of the set $\Sigma$ of singular times satisfies $d_{f}(\Sigma) \leq 1 / 2$.

Proof. Suppose that $d_{f}(\Sigma)=d>1 / 2$. Then as in the proof of Proposition 2.2 , for some $\delta$ with $1 / 2<\delta<d$ there exists a sequence $\epsilon_{j} \rightarrow 0$ such that $N_{j}=N\left(\Sigma, \epsilon_{j}\right)>\epsilon_{j}^{-\delta}$. If there centres of these balls are $t_{n}, 1 \leq n \leq N_{j}$, then since the balls are disjoint

$$
\int_{0}^{1}\|D u(s)\|^{2} \mathrm{~d} s \geq \sum_{n=1}^{N_{j}} \int_{t_{n}-\epsilon_{j}}^{t_{n}+\epsilon_{j}}\|D u(s)\|^{2} \mathrm{~d} s>\sum_{n=1}^{N_{j}} \int_{t_{n}-\epsilon_{j}}^{t_{n}}\|D u(s)\|^{2} \mathrm{~d} s .
$$

Since $\left\|D u\left(t_{n}\right)\right\|=+\infty$, the lower bound in (4) holds, and so

$$
\int_{0}^{1}\|D u(s)\|^{2} \mathrm{~d} s \geq \frac{1}{2 c} \sum_{i=1}^{N_{j}} \sqrt{c \epsilon_{j}} \geq \frac{1}{2 \sqrt{c}} \epsilon_{j}^{(1 / 2)-\delta} .
$$

Since the right-hand side tends to infinity as $j \rightarrow \infty$, we must have $d_{f}(\Sigma) \leq 1 / 2$.

Scheffer's well-known result that the 1/2-dimensional Hausdorff measure of the set of singular times is zero, $\mathscr{H}^{1 / 2}(\Sigma)=0$, is based on the same ingredients as our result, but neither is a consequence of the other. Indeed, in general the box-counting dimension gives an upper bound for the Hausdorff dimension, but from this observation the consequence of our result is only that $\mathscr{H}^{1 / 2}(\Sigma)<+\infty$. However, note that while any countable set $K$ has Hausdorff dimension zero (so in particular $\mathscr{H}^{s}(K)=0$ for all $s>0$ ), the box-counting dimension has the property that $d_{f}(X)=d_{f}(\bar{X})$, so it is easy to find a countable subset of the line with $d_{f}(X)=1$. Thus our result $d_{f}(\Sigma) \leq 1 / 2$ does indeed serve to limit further the set of singular times.

\section{Acknowledgments}

JCR is a Royal Society University Research Fellow, and would like to thank the Society for all their support. WS is currently a visiting fellow in the Mathematics Institute at the University of Warwick under the Marie Curie Host Fellowship for the Transfer of Knowledge. We would both like to thank José Rodrigo for some interesting and helpful conversations.

\section{References}

L. Caffarelli, R. Kohn, \& L. Nirenberg. Partial regularity of suitable weak solutions of the Navier-Stokes equations. Comm. Pure Appl. Math., 35: 771-831, 1982.

P. Constantin and C. Foias. Navier-Stokes Equations. University of Chicago Press, Chicago, 1988.

C.R. Doering \& J.D. Gibbon. Applied Analysis of the Navier-Stokes Equations. Cambridge Texts in Applied Mathematics, Cambridge University Press, Cambridge, 1995.

K.J. Falconer. Fractal Geometry. Wiley, Chichester, 1990.

J.A. Langa \& J.C. Robinson. Fractal dimension of a random invariant set. J. Math. Pures Appl. 85: 269-294, 2006. 
J. Leray. Essai sur le mouvement d'un fluide visqueux emplissant l'espace. Acta Math., 63: 193-248, 1934.

J.C. Robinson. Infinite-dimensional dynamical systems. Cambridge Texts in Applied Mathematics, Cambridge University Press, Cambridge, 2001.

J.C. Robinson. Regularity and singularity in the three-dimensional Navier-Stokes equations. Boletin de la Sociedad Española de Matemática Aplicada, 35: 43-71.

V. Scheffer. Turbulence and Hausdorff dimension, in Turbulence and Navier Stokes Equation, Orsay 1975, Springer LNM 565: 174-183, Springer Verlag, Berlin, 1976.

R. Temam. Navier-Stokes Equations. North Holland, Amsterdam, 1977. Reprinted by AMS Chelsea, 2001. 